\title{
Modified Viscotrabeculotomy with Microcatheter: Our Technique and Experience in the Surgical management of Glaucoma
}

\author{
Nevbahar Tamçelik ${ }^{1}$, Olgu Capar ${ }^{2}$, Eray Atalay ${ }^{3}$
}

\begin{abstract}
Aims: To present the results of our case series with a technique we defined as "modified viscotrabeculotomy" which incorporates the benefits of $360^{\circ}$ of trabeculotomy and viscodilation and viscodissection of canal of Schlemm using a microcatheter.

Materials and methods: This study was designed as a prospective interventional case series and was conducted in Cerrahpasa Medical School Ophthalmology Clinic. Forty-one eyes of 30 patients diagnosed with congenital glaucoma (CG) underwent modified viscotrabeculotomy surgery following initial preoperative examination. The main outcome measure was the surgical success of the procedure, which is determined by an intraocular pressure (IOP) of $<18 \mathrm{~mm} \mathrm{Hg}$ without medication or resurgery; qualified success is defined as an IOP of $<18 \mathrm{~mm} \mathrm{Hg}$ under general anesthesia with an additional need for medications. The differences in IOP measurements were analyzed using paired analysis of variance (ANOVA) with repeated measures.

Results: The mean preoperative IOP of the patients was $32.27 \pm 4.89 \mathrm{~mm} \mathrm{Hg}$. The IOP values at the $1 \mathrm{st}, 3 \mathrm{rd}, 6$ th postoperative months, and at the last visit were $13.41 \pm 2.25 \mathrm{~mm} \mathrm{Hg}, 13.70 \pm 1.78 \mathrm{~mm} \mathrm{Hg}, 12.47 \pm 1.57 \mathrm{~mm} \mathrm{Hg}$, and $12.26 \pm 1.81 \mathrm{~mm} \mathrm{Hg}$, respectively. The difference between the preoperative IOP values and postoperative IOP values was statistically significant $(p<0.001)$. The qualified surgical success rate was $94.4 \%$ after a mean follow-up of $27.95 \pm 8.25$ months (range 15-45 months).

Conclusion: Modified viscotrabeculotomy is a safe and effective treatment in the management of CG.

Keywords: Canaloplasty, Congenital glaucoma, Goniotomy, Microcatheter, Viscotrabeculotomy.

Journal of Current Glaucoma Practice (2019): 10.5005/jp-journals-10078-1263
\end{abstract}

\section{INTRODUCTION}

Congenital glaucoma (CG) is a relatively rare, inherited developmental disorder of the trabecular meshwork that is associated with severe visual disability if not managed promptly. Main treatment in CG is surgery, and surgical approach differs widely from that of adulthood glaucoma surgery. Defining the exact anatomic defect is the crucial step in choosing the correct treatment option. The aims of CG surgery are to decrease the localized pressure in the trabecular meshwork and to overcome the blockage of aqueous flow. Goniotomy and trabeculotomy $a b$ externo are the classic procedures that relieve the obstruction in aqueous pathway by an incision in the trabecular meshwork. ${ }^{1,2}$ It has been understood from several studies that these techniques have similar results in terms of efficacy and success rate. ${ }^{3,4}$ Recently, great efforts were made to discover the new techniques that may increase the efficacy and success rate of conventional surgeries. With the advancement of viscoelastic materials in ocular surgery, CG surgery also became a field with a potential to benefit by the use of these substances. ${ }^{4-7}$ Tamcelik previously described two surgical techniques called "viscogoniotomy" and "viscotrabeculotomy" which were found safer and more effective than their predecessors. ${ }^{5,6}$ Viscotrabeculotomy technique offers effective viscodilation and viscodissection of the canal of Schlemm using viscoelastic material with the aid of Grieshaber cannula. However, the effects of this technique is limited in extent, as the Grieshaber cannula is only able to penetrate up to $5 \mathrm{~mm}$ on either side of the canal of Schlemm. Viscodilation and viscodissection in the canal of Schlemm may be extended up to $360^{\circ}$ by the aid of a microcatheter used in canaloplasty surgery. ${ }^{7}$ This study
'Department of Ophthalmology, Istanbul University Cerrahpasa, Cerrahpasa Medical School, Istanbul, Turkey

${ }^{2}$ Department of Ophthalmology, University of Health Sciences, Okmeydani Training and Research Hospital, Istanbul, Turkey

${ }^{3}$ Department of Ophthalmology, Eskisehir Osmangazi University Medical School, Eskisehir, Turkey

Corresponding Author: Nevbahar Tamçelik, Department of Ophthalmology, Istanbul University Cerrahpasa, Cerrahpasa Medical School, Istanbul, Turkey, Phone: +90 21221551 80, e-mail: nevbahartamcelik@gmail.com

How to cite this article: Tamçelik N, Capar O, Atalay E. Modified Viscotrabeculotomy with Microcatheter:OurTechnique and Experience in the Surgical management of Glaucoma. J Curr Glaucoma Pract 2019;13(3):88-93.

Source of support: Nil

Conflict of interest: None

will give brief details about the "modified viscotrabeculotomy" technique which embraces the benefits of $360^{\circ}$ of viscodilation and viscodissection in canaloplasty surgery in combination with the conventional viscotrabeculotomy.

\section{Materials and Methods}

This study was designed as a prospective case series and was conducted in Istanbul University Cerrahpasa Ophthalmology Clinic. The study protocol adhered to the tenets of the declaration of Helsinki and Institutional Ethics Committee. The aim of this study is to evaluate 
the results of the modified viscotrabeculotomy surgery in subjects diagnosed with CG. The subjects to be studied were consecutively selected after their initial examination under general anesthesia with inhalational sevoflurane. Complete ophthalmologic examination under general anesthesia, included biomicroscopic examination, intraocular pressure (IOP) measurement by Tonopen (Mentor, Norwell, Mass), corneal diameter (CD) measurement, central corneal thickness measurement by ultrasound pachymeter (IOPac; Heidelberg Engineering, Germany), and fundoscopic examination. Forty-one eyes of 30 patients with a diagnosis of CG ( 25 eyes with primary CG and 16 eyes with secondary $(G)$ were enrolled in the study. Patients with an established diagnosis of CG underwent modified viscotrabeculotomy surgery following their initial examination.

The steps of the surgical technique are illustrated in Figure 1 and are as follows: a limbus-based conjunctival flap and a triangleshaped scleral flap were prepared in the upper temporal quadrant (Fig. 1A). Radial incision was performed until the scleral spur fibers were observed and the canal of Schlemm exposed (Fig. 1B). The canal of Schlemm was cannulated approximately $5 \mathrm{~mm}$ on either side using the Grieshaber cannula and a high-viscosity sodium hyaluronate (Healon GV ${ }^{\circledast}$; Abbott Medical Optics, Abbott Park, Illinois, USA) was injected into the canal of Schlemm while withdrawing the cannula (Figs $1 C$ and D). A canaloplasty microcatheter (iCath ${ }^{\circledast}$; iScience Interventional Corp., Menlo Park, California, USA) was prepared according to the leaflet manual. The microcatheter was introduced into the canal of Schlemm and advanced until the tip of the microcatheter exits from the other ostium (Figs $1 \mathrm{E}$ and F). The microcatheter is then withdrawn and a click of high-viscosity viscoelastic was injected at every 2 clock hours on its pathway to ensure sufficient viscodilation and viscodissection in the canal of Schlemm and trabecular meshwork, respectively (Figs $1 \mathrm{G}$ and $\mathrm{H}$ ). This procedure was repeated for either side. An angled Harms trabeculotome was inserted into either surgically created ostia of canal of Schlemm and trabeculotomy was performed (Fig. 1I). This procedure encloses approximately 20 to $30 \%$ of canal of Schlemm. Then MIOSTAT ${ }^{\circledR}$ (Carbachol Intraocular Solution, USP $0.01 \%$ ) was injected into the anterior chamber to achieve pupillary miosis. If necessary, a small amount of sodium hyaluronate (Healon ${ }^{\circledast}$; Advanced Medical Optics, Inc.) was injected into the anterior chamber to ensure anterior chamber formation. One bite of scleral tissue on the roof of the exposed canal of Schlemm was removed from either side of radial incision lips with a Luntz Dodick Glaucoma Punch with Rotatable Cutting Head (Geuder AG, G-18239) (Fig. 1J). Then a small amount of high-viscosity sodium hyaluronate was injected on the created sclera lake to prevent adhesion of incision lips. The triangular scleral flap was sutured with one 10/0 nylon suture, and the conjunctival flap was closed with a 8-0 polyglactin suture. At the end of the procedure, subconjunctival antibiotic and steroid were injected and a patch was applied on the eye.

Topical corticosteroids have been administered for up to 3 weeks until a complete resolution of inflammation was observed in the postoperative period. Topical cycloplegics were utilized 4 days after the surgery. Topical antibiotics were administered five times a day, tapered gradually over a 3-4-week period. Postoperative follow-up included complete ophthalmologic examination under general anesthesia on the first postoperative week and on the first, third, and sixth postoperative months. Patients were followed up every 3 months afterward. The fellow eyes of the patients with bilateral CG were operated 7-15 days after the first operation. Only one patient had simultaneous bilateral operation due to the risks of a second anesthesia, as advised by the pediatrician.
Preoperative and last visit IOP, corneal edema, CD, and central corneal thickness (CCT) findings were recorded from examination under general anesthesia. The degree of corneal edema was recorded as clear, mild, moderate, and severe. In clear corneas, all the details of the anterior segment could be clearly defined. Mild corneal edema was associated with a relatively hazy appearance of the cornea and anterior segment, but with no obscuration of the details. In patients with moderate corneal edema, the relatively more intense central corneal edema obscured the view of the central anterior chamber, but the peripheral iris and peripheral anterior segment details could be relatively viewed more clearly. In severe corneal edema, none of the details of the anterior segment could be examined.

Outcome measures were as follows: complete surgical success was determined by a postoperative IOP $<18 \mathrm{~mm} \mathrm{Hg}$ under general anesthesia without medication or resurgery, with no progression of optic disk cupping, no change, or a decrease in CD and clearance of preoperative corneal edema, if present. Qualified success is defined as a postoperative IOP of $<18 \mathrm{~mm} \mathrm{Hg}$ under general anesthesia, with an additional need for medications. Surgical failure was defined as an IOP $>18 \mathrm{~mm} \mathrm{Hg}$ under general anesthesia, with enlargement of $C D$ despite full medical therapy or the need for resurgery.

Collected data were recorded into a Microsoft Excel Datasheet and transferred to SPSS version 21.0 by IBM (SPSS Inc., Chicago, Illinois, USA) for statistical analysis. The differences in IOP measurements of within-subject variables were analyzed using paired analysis of variance (ANOVA) with repeated measures. The changes in preoperative and last visit CCT were analyzed using paired $t$ test. The changes in preoperative and last visit CD were analyzed using Kruskal-Wallis test. $\chi^{2}$ test was used to test the differences in preoperative and last visit corneal edema grading. A survival curve was built based on the definitions of surgical success and presented as a figure. $p$ values $<0.05$ were considered statistically significant.

\section{Results}

Of the 30 patients, 19 were male and 11 were female. All patients were under 1 year of age at first admission and at the time of operation. The mean age at the time of operation was $118.50 \pm$ 111.49 days. The mean follow-up duration was $27.95 \pm 8.25$ months (range: 15-45 months). The patients' age at the time of operation, their preoperative and postoperative findings, and their follow-up durations are shown in Table 1.

No major complications were observed postoperatively. Three patients exhibited a minor anterior chamber hemorrhage during the first postoperative week with a complete resolution in the follow-up. One eye of a patient had a temporary IOP elevation probably due to retained viscoelastics in the anterior chamber, which resolved in 3 days. All patients had an IOP below $18 \mathrm{~mm} \mathrm{Hg}$ in the last visit. Five eyes required medications (timolol maleate + dorzolamide) in the follow-up period. During the follow-up period, a reoperation was not required in any patient.

Figure 2 shows the trends of the IOP change before and after the surgery. The mean preoperative IOP of the patients was $32.27 \pm 4.89 \mathrm{~mm} \mathrm{Hg}$. The IOP values at the first, third, sixth postoperative months, and at the last visit was $13.41 \pm 2.25 \mathrm{~mm} \mathrm{Hg}$, $13.70 \pm 1.78 \mathrm{~mm} \mathrm{Hg}, 12.47 \pm 1.57 \mathrm{~mm} \mathrm{Hg}$, and $12.26 \pm 1.81 \mathrm{~mm} \mathrm{Hg}$, respectively. The difference between the preoperative and postoperative values was statistically significant $(p<0.001)$. 

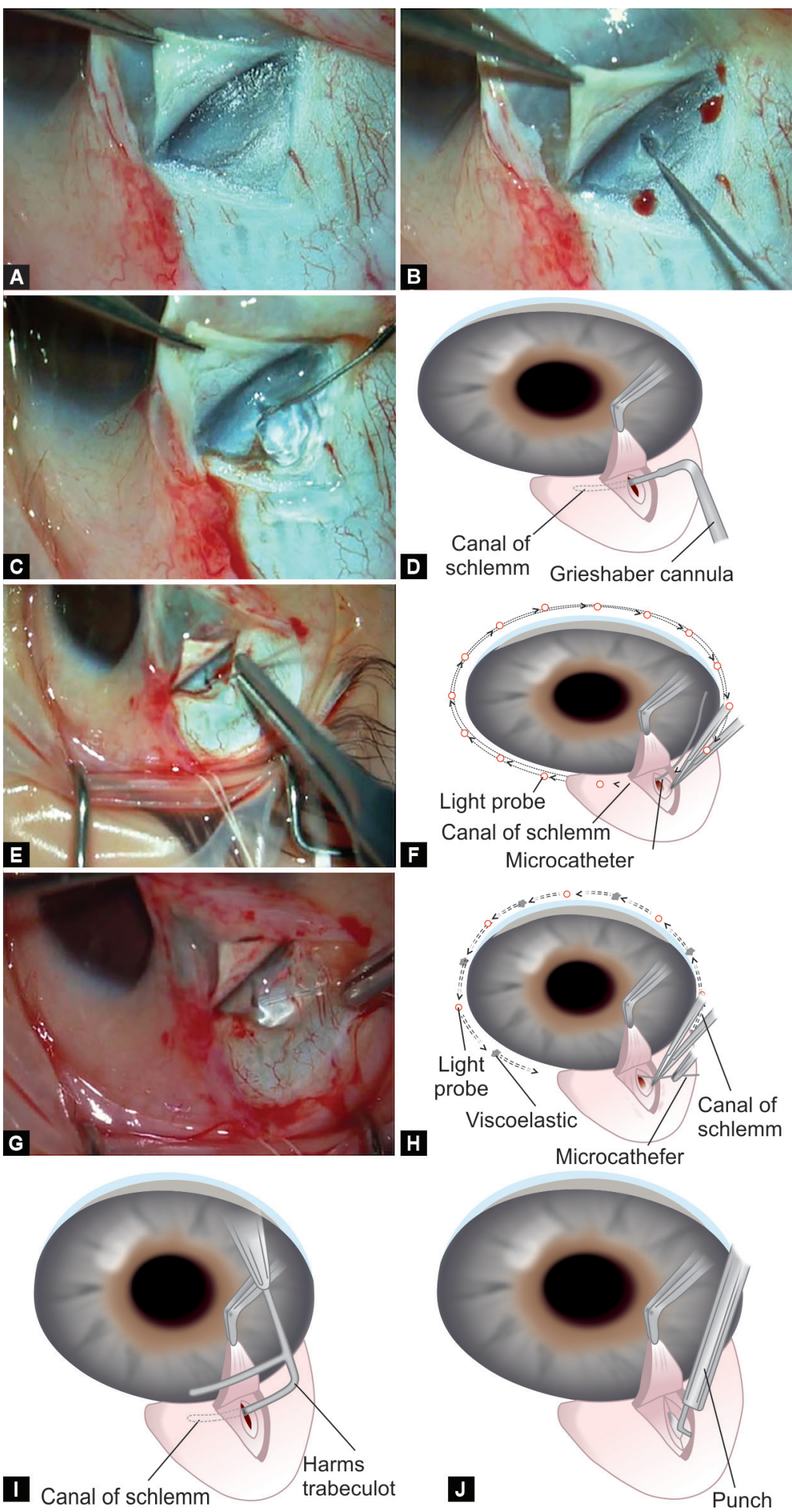

Figs 1 A to J: (A) A limbus-based conjunctival flap and a triangle-shaped scleral flap were prepared in the upper temporal quadrant; (B) Radial incision was performed until the scleral spur fibers were observed and the canal of Schlemm (SC) exposed; (C and D) The SC was cannulated approximately $5 \mathrm{~mm}$ on either side using the Grieshaber cannula and a high-viscosity sodium hyaluronate was injected into the canal while withdrawing the cannula; (E and F) A canaloplasty microcatheter (iCath ${ }^{\oplus}$; iScience Interventional Corp., Menlo Park, California, USA) was introduced into the SC and advanced until the tip of the microcatheter exits from the other ostium; $(\mathrm{G}$ and $\mathrm{H})$ The microcatheter is then withdrawn and a click of high-viscosity viscoelastic was injected at every 2 hours on its pathway; (I) An angled Harms trabeculotome was inserted into either surgically created ostia of SC and trabeculotomy was performed; (J) One bite of scleral tissue on the roof of exposed SC was removed from either side of radial incision lips with a Luntz Dodick glaucoma punch 


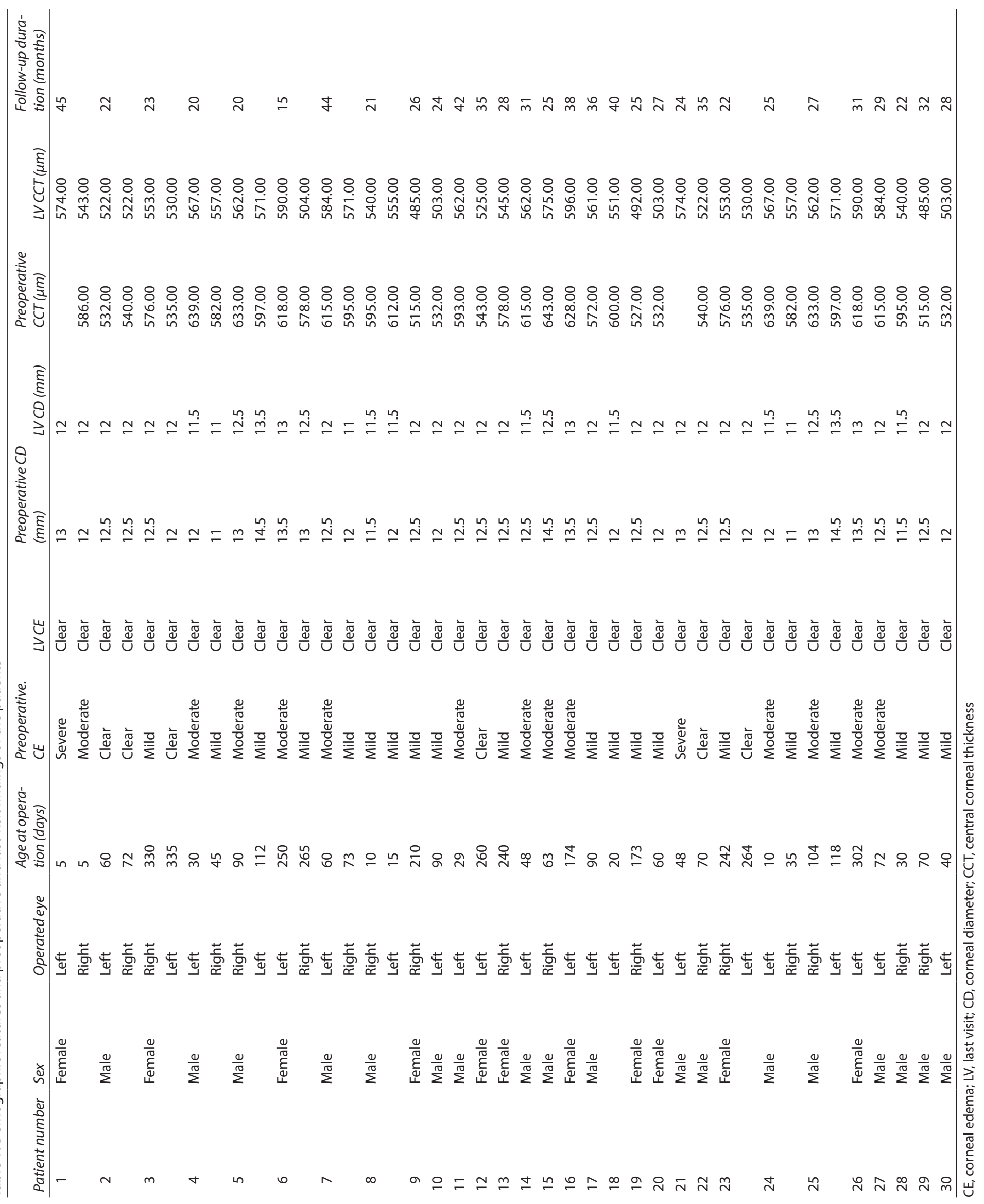




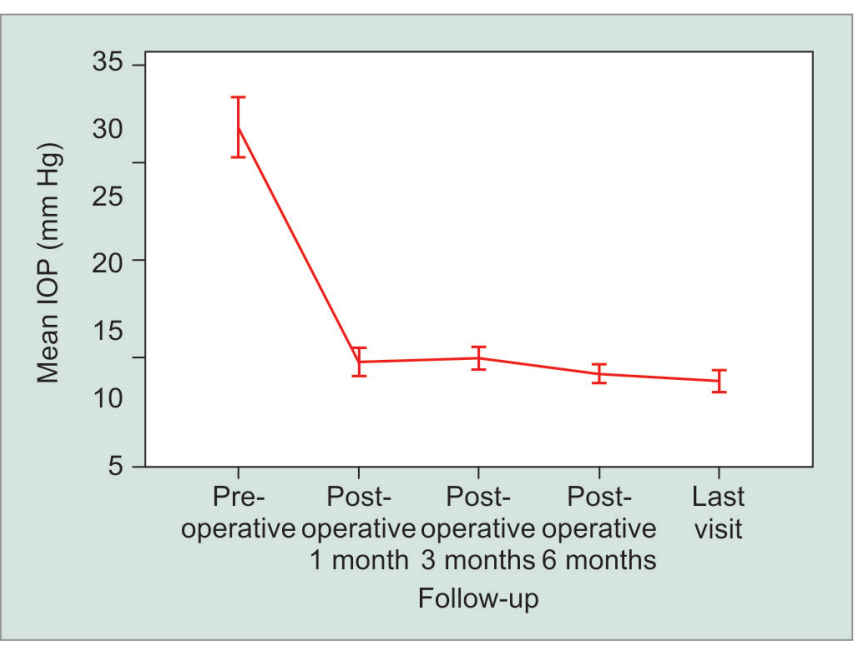

Fig. 2: Trends in intraocular pressure change during the follow-up time

The qualified surgical success rate was $94.4 \%$ after a mean follow-up of $27.95 \pm 8.25$ months. Figure 3 shows the survival curve of qualified surgical success.

The mean preoperative and last visit CCT of the patients were $581.74 \pm 38.86 \mu \mathrm{m}$ and $546.02 \pm 30.34 \mu \mathrm{m}$, respectively. The preoperative CCT of 2 eyes could not be measured due to severe preoperative corneal edema. The difference between the preoperative and postoperative values was statistically significant $(p=0.005)$. The mean preoperative CD measurements of the patients were $12.44 \pm 0.78 \mathrm{~mm}$. The mean last visit $C D$ of the patients was $12.02 \pm 0.69 \mathrm{~mm}$. The CD of the patients were lower postoperatively, and this difference was statistically significant $(p=0.049)$. The change in corneal edema was also statistically significant $(p<0.001)$. Spearman's correlation analysis showed that in the last visit, the cup-disk ratio values were significantly and positively correlated with the age at the time of operation and preoperative cup-disk ratio values ( $p<0.001, r$ : $0.584 ; p<0.001$, $r: 0.852$, respectively). Preoperative CCT was significantly and positively correlated with the last visit CCT ( $p<0.001, r$ : 0.869). No significant correlation was found between age at the time of operation and the last visit CCT ( $p$ : 0.670, $r:-0.69$ ).

\section{Discussion}

Congenital glaucoma is a relatively neglected field of ophthalmology probably owing to its low incidence in industrialized countries. ${ }^{8}$ The management of CG is surgery, which mainly focuses on strategies to relieve aqueous blockade at the level of abnormal trabecular meshwork. This can be accomplished by the incision of the meshwork $a b$ interno or ab externo with goniotomy or trabeculotomy, respectively. Although goniotomy is a preferred initial procedure with fewer complications, it is restricted to cases with no corneal edema as full visualization of the meshwork is required during the surgery. Trabeculotomy $a b$ externo is an alternative to goniotomy, which can be performed in cases with severe corneal edema in addition to cases with no corneal edema. In several studies, it has been reported that trabeculotomy has been considered as the initial procedure and is also reported to have higher success rates than goniotomy. ${ }^{1,2,9}$

With the advancement of viscoleastics in ocular surgery, Tamcelik previously defined the viscotrabeculotomy technique

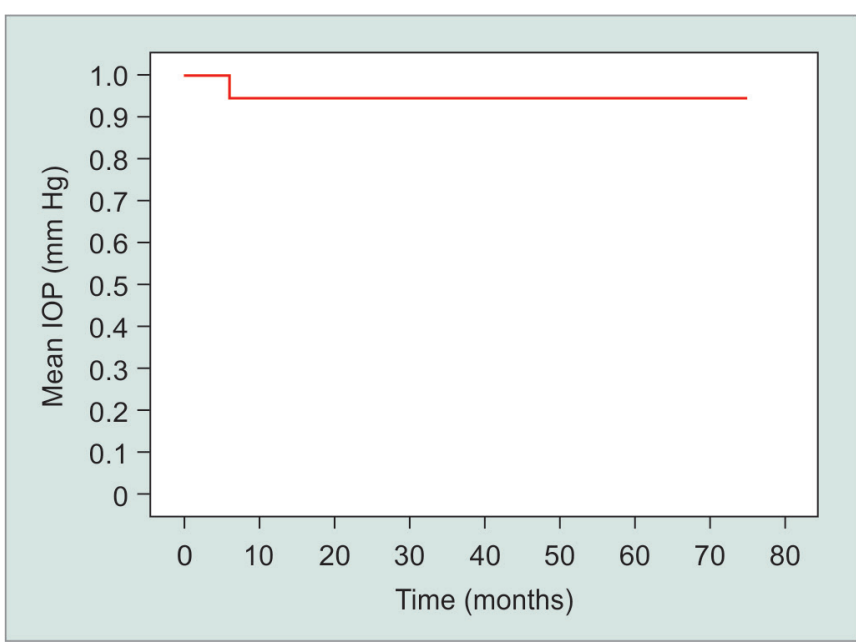

Fig. 3: Survival curve for complete surgical success over time (months)

in an attempt to render CG surgery safer and more effective than conventional methods. ${ }^{5,6}$ Conventional viscotrabeculotomy technique offers the advantage of a more easily visualized canal of Schlemm using viscoelastic materials. It provides effective viscodilation in canal of Schlemm and viscodissection of the trabecular meshwork and associated collector channels without any trauma. This viscodilation and viscodissection effect provides more enhanced outflow of aqueous humor in the canal of Schlemm compared to the classical trabeculotomy. It might be suggested that injection of viscoelastics into the canal creates microruptures in the inner wall and widening of collector channels in the outer wall of the trabeculum, allowing the aqueous to bypass the trabeculum and to enter canal of Schlemm through these microruptures. ${ }^{10}$ This might be further supported by two animal studies which have suggested that an increase in outflow facility after viscoelastic injection into the canal might be due to focal disruptions in the inner and outer endothelial walls of the canal of Schlemm. ${ }^{11,12}$ Viscodissection effect of viscotrabeculotomy surgery in CG allows the destruction of abnormal congenital tissue in the canal of Schlemm and abnormal trabecular tissue especially in the uveal trabecular meshwork. Therefore, viscodissection effect reduces the resistance and provides effective aqueous outflow. Additionally, trabecular filtration may also be enhanced by the passive pressurizing effect of viscoelastics on the trabecular meshwork in areas where trabeculotomy has not reached. Viscoelastics also prevent the collapse of the canal of Schlemm by remaining in the canal for 4-6 days. Viscoelastic materials inhibit the polymerization of fibrinogen to fibrin in the canal of Schlemm and scleral lake, limiting wound-healing process and cicatrization. ${ }^{5,6}$ It has also been speculated that the continuous presence of sodium hyaluronate in the canal of Schlemm might prevent repair of the microruptures created after viscoelastic injection by interfering with thrombocyte function. ${ }^{11}$ Viscoelastics also ensure better control of anterior chamber stability throughout the surgery. In addition, viscoelastics stop bleeding and protect the tissues during surgery, preventing any complication that might have a negative impact on the outcome. ${ }^{4,13}$

Viscodilation and viscodissection in conventional viscotrabeculotomy technique, however, are limited as the Grieshaber cannula is only able to penetrate up to $5 \mathrm{~mm}$ on either side of the canal of Schlemm. Histologic studies have 
showed that canal of Schlemm is enlarged as far as $16 \mathrm{~mm}$ downstream from the insertion point of the cannula, and this dilation decreases gradually with distance from the injection site. ${ }^{12}$ Modified viscotrabeculotomy technique enhances the efficacy of conventional viscotrabeculotomy technique by embracing the benefits of a canaloplasty microcatheter. The limited viscodissection and viscodilation of canal of Schlemm and the meshwork in the conventional viscotrabeculotomy technique is extended to $360^{\circ}$ using canaloplasty microcatheter in modified viscotrabeculotomy surgery. Moreover, removal of a bite of scleral tissue on either side of the radial incision from the roof of canal of Schlemm in modified viscotrabeculotomy technique is performed by Luntz Dodick glaucoma punch. The removal of a bite of scleral tissue creates an additional filtration surface (scleral lake), which increases the drainage of aqueous humor from the anterior chamber in addition to the dilated canal of Schlemm. This additional drainage surface does not form a bleb like in the trabeculectomy procedure because the surgically created potential space of the scleral lake is not large enough to produce a subconjunctival bleb formation. It is also relatively more posterior to the limbus and is protected by the floor of the canal of Schlemm.

An interesting finding was the significant reduction in CCT in the last visit compared to the preoperative findings. Regression analysis shows that preoperative CCT is a good predictor of the last visit CCT ( $B: 0.699, p<0.001)$. This difference might be due to higher readings of CCT preoperatively, which is related to the degree of corneal edema. Corneal diameter also decreased from baseline values and this change was nearly statistically significant. Perhaps regulation of IOP with subsequent decrease in tension on ocular tissues causes cessation of globe enlargement and reduction in $C D$.

Although the angle is avascular in primary CG, loops of vessels originating from the major arterial circle may be occasionally seen above the iris root. ${ }^{14}$ This notation carries significance, as incisional surgeries that involve the $360^{\circ}$ of the angle might end up with failure due to trauma and subsequent hemorrhage from these ambiguous vessels. Thus, modified viscotrabeculotomy might be safer than the $360^{\circ}$ trabeculotomy techniques because it does not cause gross anatomic changes in the anterior chamber angle. ${ }^{15-17}$ However, further studies are needed to assess whether this surgical technique might be of benefit in secondary CG patients who possess a higher rate of developmental ocular anomaly.

\section{References}

1. Ou Y, Caprioli J. Surgical management of pediatric glaucoma. Dev Ophthalmol 2012;50:157-172. DOI: 10.1159/000334798.
2. Ikeda H, Ishigooka $H$, Muto $T$, et al. Long-term outcome of trabeculotomy for the treatment of developmental glaucoma. Arch Ophthalmol 2004;122(8):1122-1128. DOI: 10.1001/archopht.122.8.1122.

3. Tamçelik N, Ozkiriş A. A comparison of viscogoniotomy with classical goniotomy in Turkish patients. Jpn J Ophthalmol 2004;48(4):404-407. DOI: 10.1007/s10384-004-0088-5.

4. Yu Y, Liu ZL, Cao L, et al. Clinical effect of improved viscocanalostomy for the treatment of primary congenital glaucoma. Int J Ophthalmol 2012;5(4):466-468. DOI: 10.3980/j.issn.2222-3959.2012.04.11.

5. Tamcelik N, Ozkiris A. Long-term results of viscotrabeculotomy in congenital glaucoma: comparison to classical trabeculotomy. $\mathrm{Br} J$ Ophthalmol 2008;92(1):36-39. DOI: 10.1136/bjo.2007.114389.

6. Tamcelik N, Ozkiris A, Sarici AM. Long-term results of combined viscotrabeculotomy-trabeculectomy in refractory developmental glaucoma. Eye (Lond) 2010;24(4):613-618. DOI: 10.1038/eye.2009.185.

7. Francis BA, Singh K, Lin SC, et al. Novel glaucoma procedures: a report by the American Academy of Ophthalmology. Ophthalmology 2011;118(7):1466-1480. DOI: 10.1016/j.ophtha.2011.03.028.

8. Grehn F. Congenital glaucoma surgery: a neglected field in ophthalmology? Br J Ophthalmol 2008;92(1):1-2. DOI: 10.1136/ bjo.2007.126458.

9. Meyer G, Schwenn O, Pfeiffer N, et al. Trabeculotomy in congenital glaucoma. Graefes Arch Clin Exp Ophthalmol 2000;238(3):207-213. DOI: $10.1007 / \mathrm{s} 004170050345$.

10. Razeghinejad MR, Fudemberg SJ, Spaeth GL. The changing conceptual basis of trabeculectomy: a review of past and current surgical techniques. Surv Ophthalmol 2012;57(1):1-25. DOI: 10.1016/ j.survophthal.2011.07.005.

11. Tamm ER, Carassa RG, Albert DM, et al. Viscocanalostomy in rhesus monkeys. Arch Ophthalmol 2004;122(12):1826-1838. DOI: 10.1001/ archopht.122.12.1826.

12. Smit BA, Johnstone MA. Effects of viscoelastic injection into Schlemm's canal in primate and human eyes: potential relevance to viscocanalostomy. Ophthalmology 2002;109(4):786-792. DOI: 10.1016/S0161-6420(01)01006-5.

13. Higashide T, Sugiyama K. Use of viscoelastic substance in ophthalmic surgery-focus on sodium hyaluronate. Clin Ophthalmol 2008;2(1):21-30. DOI: 10.2147/OPTH.S1439.

14. Mandal AK, Chakrabarti D. Update on congenital glaucoma. Indian J Ophthalmol 2011;59(Suppl):S148-S157. DOI: 10.4103/03014738.73683.

15. Mendicino ME, Lynch MG, Drack A, et al. Long-term surgical and visual outcomes in primary congenital glaucoma: 360 degrees trabeculotomy versus goniotomy. J AAPOS 2000;4(4):205-210. DOI: 10.1067/mpa.2000.106201.

16. Sarkisian SR. An illuminated microcatheter for 360-degree trabeculotomy [corrected] in congenital glaucoma: a retrospective case series. J AAPOS 2010;14(5):412-416. DOI: 10.1016/ j.jaapos.2010.07.010.

17. Girkin CA, Marchase N, Cogen MS. Circumferential trabeculotomy with an illuminated microcatheter in congenital glaucomas. J Glaucoma 2012;21(3):160-163. DOI: 10.1097/IJG.0b013e31822af350. 